\title{
Photogrammetric 3D Body Scanner for Low Cost Textile Mass Customization
}

\author{
Gianluca PERCOCO* and Luigi M. GALANTUCCI \\ Politecnico di Bari, Bari, Italy
}

\begin{abstract}
3D digitization of human bodies is used in several knowledge domains, as regards textile applications, the most commonly used digitizing systems can be classified on the basis of the technologies used: laser scanning, structured light projection, image elaboration and modeling, microwaves.

In the present research the authors present a properly designed low cost off-line 3D body digitizer, based on Digital Close Range Photogrammetry. Tests on dummy busts are reported, demonstrating the profitability of the technique for textile applications.
\end{abstract}

Keywords: Body Scanning, mass customization, photogrammetry.

\section{Introduction}

The realization of custom made clothes implies continuous tests, long times of delivery and often prohibitive costs. In this field one of the most interesting challenges is to automate some parts of the process to obtain a competitive product. Using Concurrent Engineering and promoting the integration between Computer Aided Design (CAD) and Computer Aided Manufacturing (CAM), it is possible to reduce the Time to market and to enhance the quality and lower the cost of completely customized garments. The first step to be completed to automate the realization of customized garment apparels is the 3D Digitization of customers. In this way Reverse Engineering (RE) can be used to achieve the Computer Aided Design (CAD) model of the customer; the CAD model can sent to the CAM interface to automatically generate paths to cut textiles and cut textiles but also to perform the virtual try-on in order to correct eventually undesired features before realization, reducing the number of tests on the customer. The virtual simulation can avoid physical Prototyping, currently used in the textile industry, with remarkable savings in terms of time and money [1]. The development of 3D CAD model reconstruction of the human body could represent a dramatic improvement towards the mass customization of clothes, accelerating e-commerce through the use of virtual mannequins [2]. Several examples are findable in literature regarding advanced methods of manufacturing clothes from $3 \mathrm{~d}$ scanned body data [3] [4] [5] [6]. In the present paper the most important 3D human body scanners, suitable for the textile industry, are listed pointing out features such as costs, ease of use, and diffusion potential. Subsequently a cost effective, photogrammetric 3D scanner, designed and realized by the authors, is proposed and tested.

\section{3D Body Scanning}

3D digitization of human bodies is used in several sectors of knowledge and can be subdivided into two main groups: a) the first group is mainly related to the visual results: cinematographic industry, computer games, art and virtual reality; b) the second group aims to the quantitative aspect of the results. As regards textile applications, the most used digitizing systems can be classified basing on the technologies used such as Laser Scanning, structured light projection, Image elaboration and modeling, microwaves. For an exhaustive analysis of several $3 \mathrm{~d}$ scanning technologies, please refer to [7].

\subsection{Laser Scanning}

Systems based on laser triangulation allow to measure three-dimensional surfaces by means of a simple apparatus constituted of laser emitter and two CCD cameras. The number of laser sources depends on the dimensions of the part of the body to be digitized. Several scanners are actually on the market for the acquisition of the entire human body, based on this technique. They differ mainly in the way of projection of the laser beam and in the way the image is captured. The majority of scanners (Cyberware, Vitronic, TecMath, Hamamatsu) projects on the body horizontal strips and takes advantage of the vertical movement of the laser head. Other typologies (Hamano Engineering) work with two rotary mirrors projecting vertical strips over the body. 
As regards scanning time and quality, it is very important [8] to block the head of the subject; in this case the scanning errors are reduced of $50 \%$ approximately, while the scanning time is reduced of about $30 \%$. The scanning time of commercial laser scanners can vary between 5 and 20 seconds. Medical scanners have much better performances than those requested by the apparel industry, achieving an accuracy approximately equal to 0.2 millimeters and acquisition time approximately equal to 0.3 seconds. The costs of these technologies are still very high and this is reflected in the selling price of the body scanners, that can reach several hundreds of thousands Euros. The high price of this type of scanner is a strong disadvantage, as it limits very much the diffusion of this technology in the textile field.

\subsection{Structured light projection}

If compared to laser scanning, structured light allows to acquire the shape in one step only, with a remarkable saving in terms of time. Indeed the laser scanning asks 5 to 20 seconds, while the projection of strips employs about one second. The advantage of the short scanning time resides in the reduction of errors related to uncontrolled movements of the subject. On the other hand when the surface to be scanned is wide, it is necessary to employ several units, constituted by light projector and CCD sensors, in order to correctly work. Interferences among them must be avoided activating serially the projectors, increasing the scanning time. Another possible solution is to increase the distance between the subject and the projector; in this case the overall dimensions of the system is very high. Such scanners are competing with laser, also because natural light is safer than laser. Other advantages are the low sensitivity to colors, ease of use, easy maintenance due to the absence of moving parts.

\subsection{Image Processing}

In this section, passive image processing techniques are described to achieve 3D dimensional models of the Human body, only using digital images.

\subsubsection{Silhouettes extraction}

The silhouettes extraction is based on a set of images of the person using several cameras, a single rotary camera or a single camera and a rotary platform. The images are subsequently processed to extract bi-dimensional profile that combined lead to the three-dimensional model [9]. Particularly, the volumetric representation of the human body turns out from the intersection of the visual cones obtained projecting each silhouette towards the corresponding point of view [10]. This method can achieve unconnected volumes or protrusion not correspondent to real part of the body, due to the high complexity of the geometrical shape.

\subsubsection{Photogrammetry}

Instantaneous 3-D imaging systems, based on photogrammetry, where complete 3-D information are gained with the acquisition and matching of several images. These methods are particularly suited for the digitization of human body data because of their insensitivity to slight body movements. In order to create a model of the body, the 3-D information can be directly acquired or a generic pre-existing model of the face may be employed, then adapted to the specific person. A process based on photogrammetry consists of five steps: (a) acquisition of images from different directions; (b) determination of the camera positions; (c) establishment of a dense set of corresponding points in the images; (d) computation of their 3-D coordinates; (e) generation of a surface model. One of the most critical aspects of this technique is camera calibration, that is the process of determining the characteristics of cameras to be used as measurement devices.

As regards step (b) parameters such as the focal length of the lens, the digitizing scale (which is the CCD format size of a scanner or digital camera) and the main point (where the optical axis of the lens intersects the photograph) are needed. To get improved accuracy, parameters that describe the distortion characteristics of the lens are used too.

As regards steps (c) and (d), a complete reconstruction of the human body is possible through the process of points matching between several images, basing on the least-squares method (LSM) and on the exploitation of epipolar geometry. Photogrammetry does not find important applications related to the scanning of human body, actually performed by means of laser or structured light based scanners [11]. On the other hand the costs of such scanners are still high and they do not facilitate the capillary spreading of these useful technologies, photogrammetry would introduce the advantage to lower costs dramatically. 


\subsection{Microwaves Body Scanner}

One of the most recent systems is based on millimeter-waves scanning. It exploits low power electromagnetic waves, defined millimeter-waves, in order to create a holographic 3D image of the human body. Microwaves frequency varies between 1 and $30 \mathrm{GHz}$, the millimeter-waves are between 30 and $300 \mathrm{GHz}$; they both are exploitable for anthropometric applications due to their biocompatibility when low power levels are used. The process lasts about 10 seconds digitizing hundreds of thousand points and its accuracy is about 6 millimeters. However disadvantages are represented by the low accuracy of the measurement, moreover people could be reluctant to be crossed by radio waves. Moreover also in this case costs are very high. According to the authors, the photogrammetric technique introduce the best margins of improvement, owning intrinsic characteristics such as very low scanning times, low cost equipment and no intrusiveness.

\section{Experimental setup}

In this paper it is proposed one specifically designed photogrammetric 3D scanner (Fig. 1) for the human body, focused on the automatization of the image elaboration procedures. The scanning system is composed of 8 usb video cameras with resolution equal to 5 Mpixels, equipped with $16 \mathrm{~mm}$ wide lenses ; 4 white light illuminators, $100 \mathrm{~W}$ each. The elaboration of the images has been carried out through the software Photomodeler 6.0 by Eos Systems. The calibration of the system has been performed using one $12 \times 12$ points grid with 4 coded points. Six images per camera have been used, for a total of 48 photos.

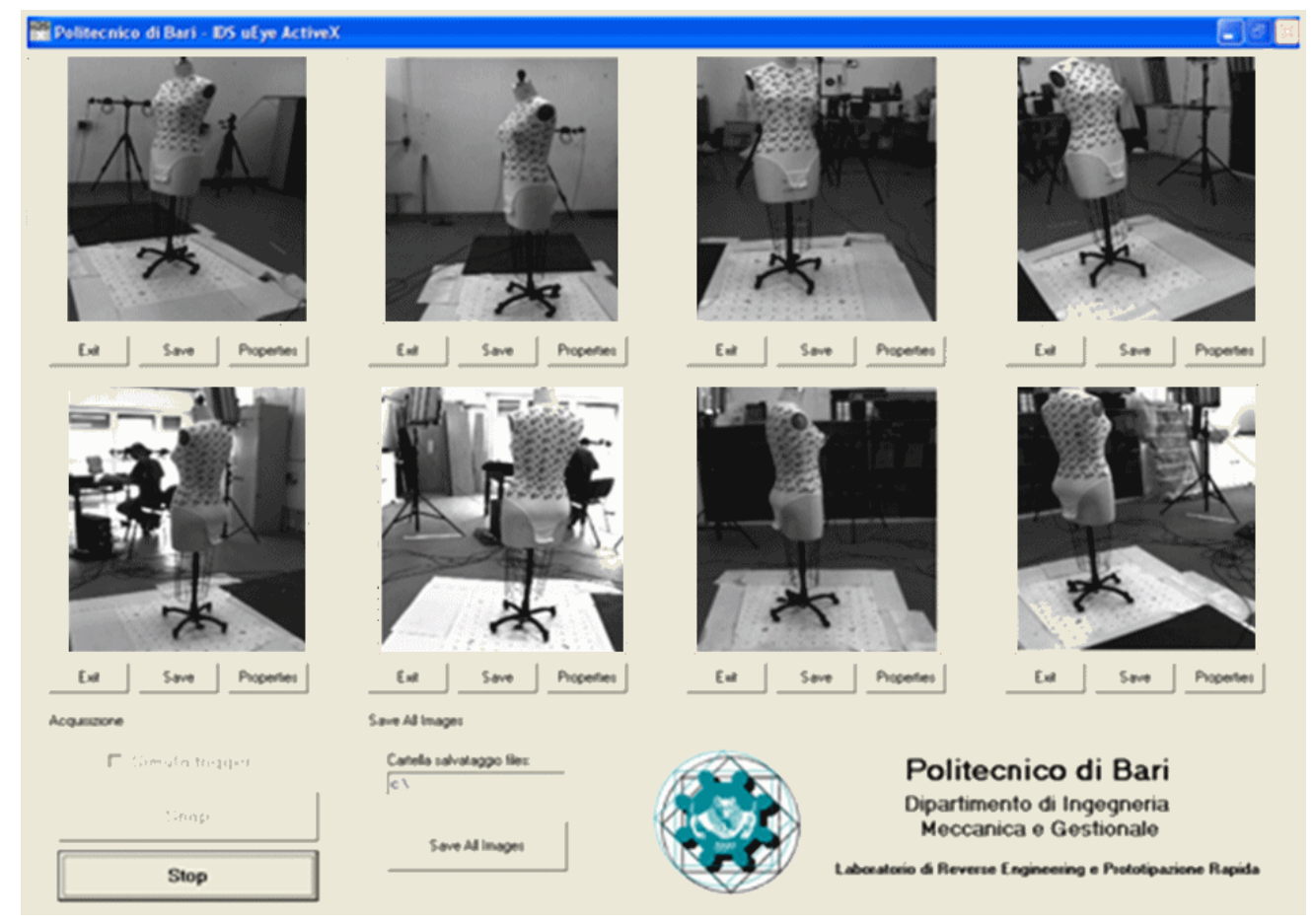

Fig. 1: Software Interface for image acquisition

Coded Targets are circular targets with additional bits that can be automatically recognized by the software during the marking process (Fig. 2). This automatic recognition allows for automated referencing even before a project has been processed and in complex scene or shape. Coded Targets increase the speed at which projects can be completed, improve the speed and robustness of the crucial orientation stage which all projects must go through at the start, and improve robustness and reduce the chances of misreferenced points. The dimensions of the coded targets are very important to have a correct referencing and digitization. 


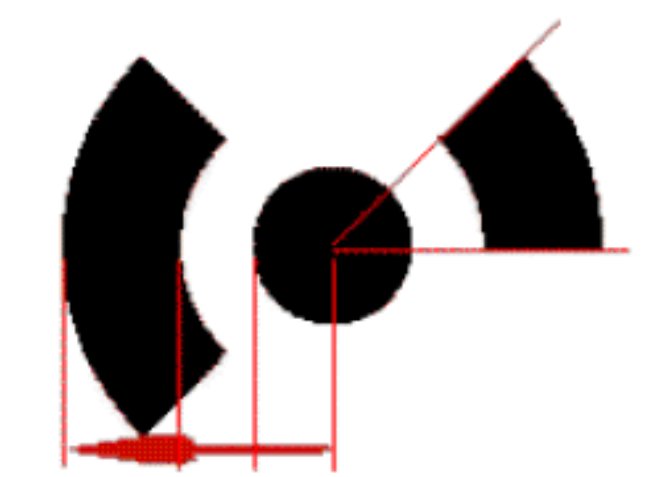

\section{Bit Code}

Re Rs Rt

Fig. 2: Example of coded target

\section{Results}

The scanner have been preliminarily validated through several tests, described in the present paper, namely tests on dummy busts, with an Italian size 42 (correspondent to an American size 8). The busts have been dressed with specially designed corsets (Fig. 3:). As regards the corsets, several tests have been conducted. For the sake of brevity in the present paper three different typologies are reported: (i) coded targets with Re equal to $3,5 \mathrm{~cm}$ and Rt equal to $1 \mathrm{~cm}$; (ii) hybrid coded-non coded targets where the coded targets are equal to case (i) and non coded targets with diameter equal to $1 \mathrm{~cm}$; (iii) hybrid coded-non coded targets where the coded targets are equal to case (i) and (ii) and non coded targets diameter is equal to $0.5 \mathrm{~cm}$.

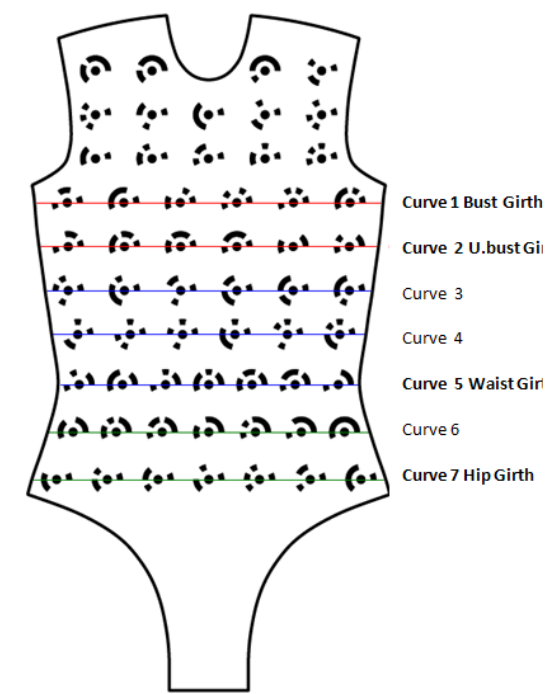

Fig. 3: (i) coded targets

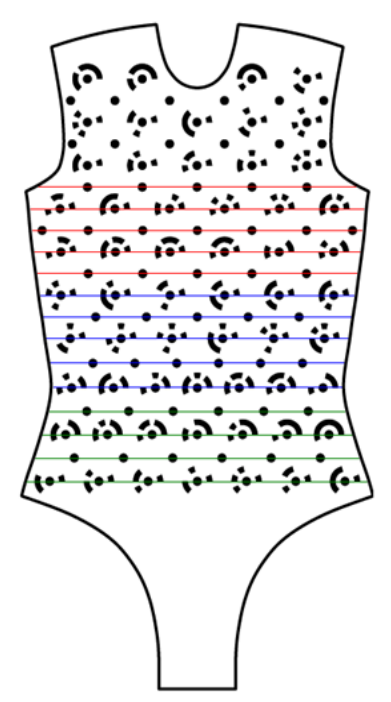

(ii) hybrid, with larger non coded targets

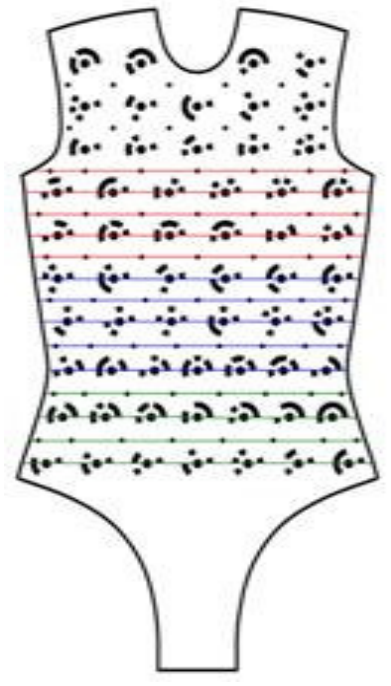

(iii) hybrid, smaller non coded targets

The corset (i) allows to measure seven curves from the bust to the waist line. The use of non-coded targets allows to double the digitizable curves and to digitize up to 200 points to detail better the CAD model. The measurable curves are pointed out in Fig. 3 for the three corsets; in bold the curves cited by the norm EN 13402, the European standard for labelling clothes sizes. In Fig. 4 a tessellated model of the dummy bust is used as basis for comparison with tessellated models obtained by the three corsets. It is possible to notice how the presence of non-coded points improves accuracy and this phenomenon is more evident with smaller non-coded targets. 


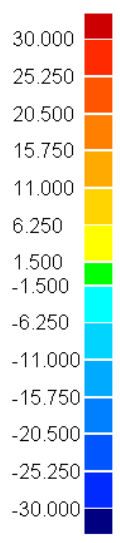

Fig. 4:

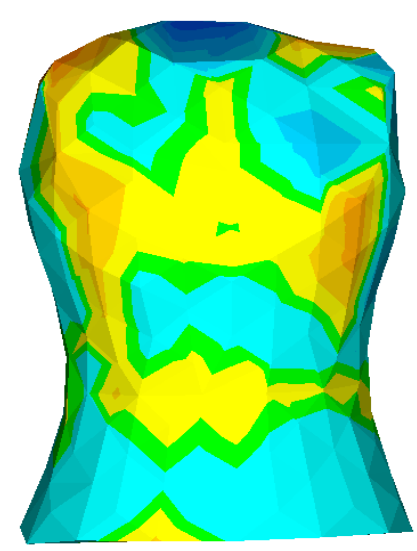

Digitised curves with corset (i)

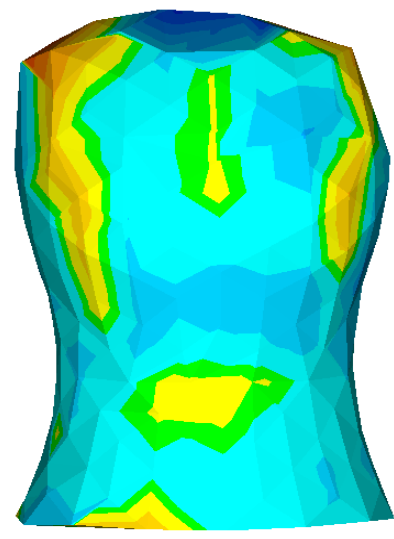

Digitised curves with corset (ii)

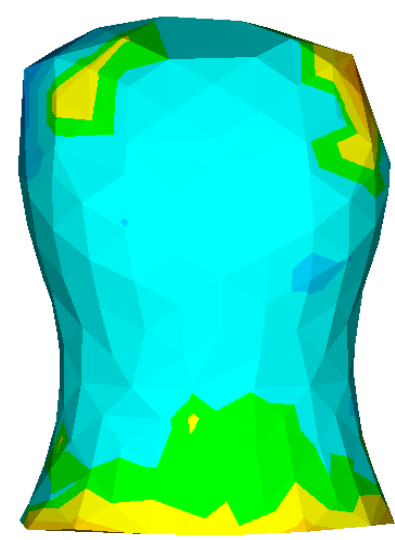

Digitised curves with corset (iii)

Each curve has been measured using a classical tape-measure and the 3D digitizing system designed by the authors. As regards the latter systems the curves have been obtained interpolating with a commercial 3D CAD software the points.

The results are shown in Tables 1,2 and 3, respectively related to measures performed on the tailor's dummy with corsets (i), (ii) and (iii)

Table 1 Measurement on tailor's dummy with corset (i)

\begin{tabular}{|l|l|l|l|l|}
\hline Measured Sizes & $\begin{array}{l}\text { Tailor measure } \\
(\mathrm{cm})\end{array}$ & $\begin{array}{l}\text { Photogrammetric Measure } \\
(\mathrm{cm})\end{array}$ & $\begin{array}{l}\text { Difference } \\
(\mathrm{cm})\end{array}$ & $\begin{array}{l}\text { Difference } \\
\%\end{array}$ \\
\hline Curve 1 Bust Girth & 95,9 & 96,04 & $-0,14$ & $-0,15$ \\
\hline Curve 2 Underbust Girth & 93,3 & 92,97 & 0,33 & 0,35 \\
\hline Curve 3 & 82,7 & 83,5 & $-0,8$ & $-0,97$ \\
\hline Curve 4 & 76,5 & 76,97 & $-0,47$ & $-0,61$ \\
\hline Curve 5 Waist Girth & 76,3 & 76,08 & 0,22 & 0,29 \\
\hline Curve 6 & 82,7 & 83,2 & $-0,5$ & $-0,60$ \\
\hline Curve 7 Hip Girth & 91,5 & 91,85 & $-0,35$ & $-0,38$ \\
\hline
\end{tabular}

The difference measured has been computed as the average absolute difference between manual and digital measures. As regards corset (i) it has been equal to 0,48 \%, satisfying for tailoring applications. The highlighted curves are from the above mentioned UNI-EN standard. The hybrid corset (ii) (Table 2) introduces a an average difference approximately equal to $0,42 \%$ for 212 digitized points; however this corset introduces also difficulties related to target marking during the image elaboration phase due to the dimension of uncoded targets very similar to that of the ring codes, needing an increase of human intervention.

Table 2 Measurement on tailor's dummy with corset (ii)

\begin{tabular}{|l|l|l|l|l|}
\hline Measured Sizes & $\begin{array}{l}\text { Tailor measure } \\
(\mathrm{cm})\end{array}$ & $\begin{array}{l}\text { Photogrammetric Measure } \\
(\mathrm{cm})\end{array}$ & $\begin{array}{l}\text { Difference } \\
(\mathrm{cm})\end{array}$ & Difference \% \\
\hline Curve 1 & 94,1 & 94,28 & $-0,18$ & $-0,19$ \\
\hline Curve 2 Bust Girth & 96 & 95,55 & 0,45 & 0,47 \\
\hline Curve 3 & 95,5 & 95,2 & 0,3 & 0,31 \\
\hline Curve 4 Underbust Girth & 91,1 & 91,14 & $-0,04$ & $-0,04$ \\
\hline Curve 5 & 86 & 86,05 & $-0,05$ & $-0,06$ \\
\hline Curve 6 & 81,2 & 81,44 & $-0,24$ & $-0,30$ \\
\hline Curve 7 & 77,2 & 77,85 & $-0,65$ & $-0,84$ \\
\hline Curve 8 & 75,5 & 76,25 & $-0,75$ & $-0,99$ \\
\hline Curve 9 & 74,6 & 75,11 & $-0,51$ & $-0,68$ \\
\hline Curve 10 Waist Girth & 76,2 & 76,67 & $-0,47$ & $-0,62$ \\
\hline Curve 11 & 79,4 & 79,76 & $-0,36$ & $-0,45$ \\
\hline Curve 12 Over Hip Girth & 85 & 84,92 & 0,08 & 0,09 \\
\hline Curve 13 & 89,4 & 89,18 & 0,22 & 0,25 \\
\hline Curve 14 Hip Girth & 92,7 & 93,26 & $-0,56$ & $-0,60$ \\
\hline
\end{tabular}


The results related to corset (iii) are shown in Table 3 with an average error on the measures equal to $0,49 \%$ measured on 225 digitized points. With this corset the marking and referencing phases were faster and more points were digitized thanks to the smaller dimensions on uncoded targets.

Table 3 Measurement on tailor's dummy with corset (iii)

\begin{tabular}{|c|c|c|c|c|}
\hline Measured Sizes & $\begin{array}{l}\text { Tailor measure } \\
(\mathrm{cm})\end{array}$ & $\begin{array}{l}\text { Photogrammetric Measure } \\
(\mathrm{cm})\end{array}$ & $\begin{array}{l}\text { Difference } \\
(\mathrm{cm})\end{array}$ & Difference \% \\
\hline Curve 1 & 94,6 & 94,93 & $-0,33$ & $-0,35$ \\
\hline Curve 2 Bust Girth & 95,6 & 95,84 & $-0,24$ & $-0,25$ \\
\hline Curve 3 & 96,3 & 96,42 & $-0,12$ & $-0,12$ \\
\hline Curve 4 Underbust Girth & 92,8 & 92,67 & 0,13 & 0,14 \\
\hline Curve 5 & 87,2 & 86,95 & 0,25 & 0,29 \\
\hline Curve 6 & 82,7 & 83,45 & $-0,75$ & $-0,91$ \\
\hline Curve 7 & 78,8 & 79,6 & $-0,8$ & $-1,02$ \\
\hline Curve 8 & 77,2 & 77,69 & $-0,49$ & $-0,63$ \\
\hline Curve 9 & 75,5 & 76,31 & $-0,81$ & $-1,07$ \\
\hline Curve 10 Waist Girth & 76,5 & 77,03 & $-0,53$ & $-0,69$ \\
\hline Curve 11 & 79,3 & 79,56 & $-0,26$ & $-0,33$ \\
\hline Curve 12 Over Hip Girth & 84,3 & 83,96 & 0,34 & 0,40 \\
\hline Curve 13 & 89,2 & 89,63 & $-0,43$ & $-0,48$ \\
\hline Curve 14 Hip Girth & 92,5 & 92,68 & $-0,18$ & $-0,19$ \\
\hline
\end{tabular}

\section{Conclusions}

The experimental 3D scanner presented in this paper confirms the usefulness of photogrammetry with coded targets for digitalization of human bodies. The three-dimensional information regarding the dummy bust is correctly retrieved. In particular tailor measures can be obtained thanks to properly designed corsets. In order to increase the accuracy of the process, further studies will be focused on humans and dense surface models.

\section{Acknowledgements}

The present work has been funded by Regione Puglia, Research Project PE 014, 2007 "Development of a prototype of an anthropometric chamber for $3 \mathrm{D}$ scanning of the human body using digital close range photogrammetry for custom made garments.".

The authors wish to thank Dr. Michael De Robertis and Dr. Antonio Chiarella for their precious work in setting up the scanner designed by the authors.

\section{Bibliography}

1. L. Chittaro, D. Corvaglia, 3D Virtual Clothing: from Garment Design to Web3D Visualization and Simulation, pubblicato in: Proceedings of Web3D 2003, 8th International Conference on 3D Web Technology, ACM Press, New York, March 2003, pp. 73-84.

2. Frédéric Cordier, WonSook Lee, HyeWon Seo, Nadia Magnenat-Thalmann, From 2D Photos of Yourself to Virtual Try-On Dress on the Web, MIRALab, University of Geneva, http://tclab.kaist.ac.kr/ fcordier/Home_files/Papers/From_2D_photos_of_yourself_to_virtual_try-o n_dress_on_the_web.pdf, last access on May, the 18th 2009.

3. Sungmin Kim, Chang Kyu Park: "Basic garment pattern generation using geometric modeling method", International Journal of Clothing Science and Technology, Vol. 19 No. 1, 2007, pp. 7-17

4. Au, C., Yuen, M. (1999), "Feature-based reverse engineering of mannequin for garment design", Computer-Aided Design, Vol. 15 No.1, pp.751-9.

5. Kim, S., Kang, T. (2002), "Garment pattern generation from body scan data", Computer-Aided Design, Vol. 35 pp.611-8 
6. Kang T. J, Kim S. M: "Optimized garment pattern generation based on three-dimensional anthropometric measurement", International Journal of Clothing Science and Technology, Vol. 12 No. 4, 2000, pp. 240-254

7. www.homometrica.ch/, last access on May, the 18th 2009

8. H.A.M. Daanen, M.A. Brunsman, K.M. Robinette, Reducing movement artifacts in whole body scanning, pubblicato in: Proceedings of the international conference on recent advances in 3D digital imaging and modelling, Ottawa, Ontario, Canada, 1997, pp. 262-265.

9. Nicola D'Apuzzo, Surface measurement and tracking of human body parts from multi-image video sequences, ISPRS journal of photogrammetry and remote sensing ISSN 0924-2716, 2002, vol. 56, no 5-6, pp. 360-375, 2002.

10. Andrea Bottino, Aldo Laurentini, A Silhouette Based Technique for the Reconstruction of Human Movement, $2000 \mathrm{http}: / / w w w 2 . p o l i t o . i t / r i c e r c a / c g v g / A r t i c l e s / C V I U 2001 . p d f$, last access on May, the 18th 2009

11. K. P. Simmons, Body measurement techniques: a comparison of three-dimensional body scanning and physical antrophometric methods, Raleigh, North Carolina, 2001, http://www.tx.ncsu.edu/3dbodyscan/pdf_docs/microsoft\%20word\%20-\%20a1paper2.pdf , last access on May, the 18th 2009. 